\title{
D-bound and the Bekenstein bound for the surrounded Vaidya black hole
}

\author{
H. Hadi ${ }^{1, \mathrm{a}}$, F. Darabi ${ }^{1, \mathrm{~b}}$, K. Atazadeh ${ }^{1, \mathrm{c}}$, Y. Heydarzade ${ }^{2, \mathrm{~d}}$ \\ ${ }^{1}$ Department of Physics, Azarbaijan Shahid Madani University, Tabriz 53714-161, Iran \\ ${ }^{2}$ Department of Mathematics, Faculty of Sciences, Bilkent University, 06800 Ankara, Turkey
}

Received: 11 August 2020 / Accepted: 23 November 2020 / Published online: 7 December 2020

(C) The Author(s) 2020

\begin{abstract}
We study the Vaidya black hole surrounded by the exotic quintessence-like, phantom-like and cosmological constant-like fields by means of entropic considerations. Explicitly, we show that for this thermodynamical system, the requirement of the identification of the D-bound and Bekenstein entropy bound can be considered as a thermodynamical criterion by which one can rule out the quintessence-like and phantom-like fields, and prefer the cosmological constant as a viable cosmological field.
\end{abstract}

\section{Introduction}

The simplest candidate for dark energy in the context of an accelerating universe is the cosmological constant. The relevant cosmological model including the cosmological constant is the so-called $\Lambda$ cold dark matter $(\Lambda \mathrm{CDM})$ model, which is consistent with current observations. However, $\Lambda \mathrm{CDM}$ suffers from two well-known problems, namely the coincidence problem [1-3] and the cosmological constant problem [4]. To resolve these problems, some exotic models of dark energy, like quintessence and phantom fields, have been introduced. The quintessence field with a dynamical equation of state, having the negative equation of state parameter $-1<\omega_{q}<-\frac{1}{3}$, is capable of describing the latetime cosmic acceleration [5]. The quintessence field plays an important role in the cosmological dynamics including matter and radiation [6-9]. The phantom field, as another candidate for dark energy, has also a negative equation of state parameter $\omega_{p}<-1$, and it also is capable of describing the current acceleration of the universe [10-12]. In the limit of $\omega_{p}$ approaching a constant value, a big-rip singular-

\footnotetext{
a e-mail: hamedhadi1388@gmail.com

be-mail: f.darabi@azaruniv.ac.ir

c e-mail: atazadeh@azaruniv.ac.ir (corresponding author)

de-mail: yheydarzade@bilkent.edu.tr
}

ity results as a new problem. Having these three models of dark energy that are successful in predicting an accelerating cosmic dynamics does not imply their perfect description of the current accelerating universe and one is highly motivated to revisit these three dark energy models from a non-cosmic point of view.

The powerful thermodynamical approach and the relevant entropic limits can be considered as such non-cosmic point of view in the study of dark energy models. In principle, the equations of motion can perfectly predict the dynamical behavior of time-reversible physical systems; however, in reality, for thermodynamical systems the time-reversibility is not observed because of the entropic considerations. Dynamical black holes, surrounded by the cosmological fields, are relevant examples of such a thermodynamical system, in the present study. Explicitly, we impose an entropic criterion on the Vaidya black hole surrounded by some exotic fields possessing an average equation of state like the quintessence, phantom and cosmological constant. By means of this criterion, we investigate which of these fields can be singled out as the most viable cosmological field.

In the 1970s, the quantum physics of black holes started by the work of Bekenstein [13,14] and Hawking [15]. There is a general conviction that Hawking radiation [15] and Bekenstein-Hawking entropy $[13,15]$ are the main features of a yet unknown theory of quantum gravity which will be able to unify Einstein's general theory of relativity with quantum mechanics. In fact, some of the experts on quantum gravity claim that black holes are the fundamental bricks of quantum gravity which play the same role as atoms in quantum mechanics [16]. In this framework, Bekenstein has found a fundamental result indicating the maximum entropy of the black hole which is allowed by quantum theory and general theory of relativity [17] for a given mass and size. The Bekenstein bound puts an upper bound on the entropy of the system with a finite amount of energy and a given size. This bound is the maximum amount of information 
required to describe a system by considering its quantum properties [17]. If the energy and size of the system is finite, the information required to describe it completely is finite too. One of the important consequences of the Bekenstein bound is in the physics of information and in computer science when it is connected with the so-called Bremermann limit [18]. It sets a maximum information-processing rate for a system with finite size and energy. Another consequence of the Bekenstein bound is the derivation of the field equations of general theory of relativity [19]. There are some investigations in trying to find some forms of the bound by considering consistency of the laws of thermodynamics with the general theory of relativity [20]. In this framework, a generalization of the Bekenstein bound was derived by Bousso [21], conjecturing an entropy bound with statistical origin which is valid in all space-times consistent with Einstein's equations. This so-called covariant entropy bound reduces to the Bekenstein bound in a system of limited self-gravity [21]. Another attempt in this regard has been made by Bousso in considering the systems with a cosmological horizon which has led to the so-called D-bound [22]. Bousso has derived the D-bound for an asymptotically non-flat Schwarzschild-de Sitter black hole solution. One can look for the D-bound for other solutions which are not asymptotically flat and include a cosmological apparent horizon.

Surrounded Vaidya black holes, as asymptotically non-flat solutions, show interesting results under consideration of the D-bound which we intend to study in this paper. In fact, the Vaidya solution provides a non-static solution for the Einstein field equations which is a generalization of the static Schwarzschild black hole solution. This solution depends on the dynamical mass $m=m(u)$ as a function of the retarded time coordinate $u$, and an ingoing/outgoing flow $\sigma(u, r)$. Because of this feature of the Vaidya solution, it can be considered as a classical model for a dynamical black hole which is effectively evaporating or accreting. The process of spherical symmetric gravitational collapse has also been studied by applying the Vaidya solution. On the other hand, this solution is a testing ground for the cosmic censorship conjecture [23-26]; see also [27] for other applications.

Bousso has considered the D-bound and the Bekenstein bound for the stationary Schwarzschild-de Sitter solution and found that these two bounds are identified for this specific solution [21]. The Vaidya black hole surrounded by cosmological fields [28] is a generalization of the stationary Schwarzschild-de Sitter black hole to the case of a dynamical black hole embedded in a dynamical background. Therefore, from the entropic point of view, this generalization can account for the dependence of the D-bound and the Bekenstein bound on the dynamics of a black hole and its sur- rounding fields. ${ }^{1}$ In this paper, motivated by Bousso's work [21] on a stationary black hole solution, we show that the identification of a generalized D-bound and the Bekenstein bound for a dynamical black hole solution surrounded by some exotic cosmological fields can be considered as a suitable criterion for selecting the cosmological constant-like field as the most viable field and ruling out other fields such as phantom-like and quintessence-like fields. It is worth to mention that the physical motivation for requiring the identifications of these entropy bounds is that both of them are direct results of the generalized second law of thermodynamics putting an upper bound on the same matter system.

The organization of the paper is as follows. In Sect. 2, we review very briefly the D-bound and the Bekenstein bound. In Sect. 3, we introduce the Vaidya black hole solution. In Sect. 4, we derive the D-bound and the Bekenstein bound for a Vaidya solution with surrounding cosmological constantlike field. In Sects. 5 and 6, these bounds are obtained for the surrounded Vaidya solution by a quintessence-like field and a phantom-like field, respectively. Finally, we draw our a conclusion in Sect. 7.

\section{Bekenstein bound and D-bound}

In this section, we study the Bekenstein bound and the Dbound and summarize the important results obtained in [22] (for more details see [13,17,29]).

The Bekenstein bound is expressed by the following statement: isolated, stable thermodynamic systems in asymptotically flat space are constrained by the universal entropy bound

$S_{m} \leqslant 2 \pi R E$,

where $R$ is the radius of the circumscribing sphere system and $E$ its total energy. The Bekenstein bound has been considered in two forms, empirical and logical.

- Empirical form: All physically reasonable, weakly gravitating matter systems satisfy the Bekenstein bound $[29,30]$. Some of the systems saturate the bound. For example, the bound is saturated by a Schwarzschild black hole through $S=\pi R^{2}$ and $R=2 E$. It seems that the Bekenstein bound is the tightest one for any physical

\footnotetext{
${ }^{1}$ For non-static systems, as well as static systems, surrounded by cosmological fields, the D-bound and the Bekenstein bound can be implemented because both of them are a direct consequence of generalized second law of thermodynamics (GSL). GSL has been proven for semiclassical quantum fields (rapidly changing with time while falling across a causal horizon) minimally coupled to general relativity [52]. Moreover, GSL holds on any causal horizon [60].
} 
system. There are some controversial examples which claim the violation of the Bekenstein bound [31]. However, some of these counter-examples are shown not to correctly include the whole of the gravitating matter system in $E$ and including them can restore the Bekenstein bound. The rest of the counter-examples also contain controversial matter and excluding them from $E$ can restore the Bekenstein bound [32,33].

- Logical form: Bekenstein has claimed that, for weakly gravitating systems, the bound is a result of the generalized second law of thermodynamics (GSL) [13,17,34, 35]. By the Geroch process (a gedankenexperiment), the system is collapsed into a large black hole. The entropy of the system (black hole) becomes $\triangle A / 4=8 \pi R E$. According to GSL, $\Delta A / 4-S_{m} \geqslant 0$ where $S_{m}$ is the entropy of the lost matter system before the formation of a black hole. There are also some controversial arguments as to whether one can derive the Bekenstein bound by a Geroch process considering quantum effects [36-38]. However, there is no certain result coming out of these arguments.

The D-bound is expressed by the following statement: a D-bound is a bound on the entropy of matter systems in de Sitter space which is shown to be closely related to the Bekenstein bound in a flat background [22]. The definition of the D-bound on matter entropy in de Sitter space is as follows. Assume an observer located within his apparent cosmological horizon corresponding to a matter system, in a universe that is asymptotically de Sitter in the future. The observer moves relative to the matter until the matter is located at his apparent cosmological horizon. He will realize that crossing out of the matter from his apparent cosmological horizon is a thermodynamic process. The entropy of the system after the matter is crossed out the cosmological horizon is

$S_{0}=\frac{A_{0}}{4}$

where $A_{0}$ is the area of cosmological horizon given by

$A_{0}=\pi r_{0}^{2}=\frac{12 \pi}{\Lambda}$.

The entropy of the initial state is the sum of the matter system's entropy $S_{m}$ and a quarter of the apparent cosmological horizon

$S=S_{m}+\frac{A_{c}}{4}$.

According to the generalized second law of thermodynamics, the observer concludes that the entropy increases. Thus, by comparing Eqs. (2) and (4) we have

$S_{m} \leqslant \frac{1}{4}\left(A_{0}-A_{c}\right)$, which is the D-bound on the matter system in asymptotically de Sitter space. The D-bound has been derived by Bousso for the entropy of the matter systems in de Sitter space. It is indicated that the D-bound is the same as the Bekenstein bound of the system in this model. Also, Bousso has achieved the same result for arbitrary dimensions. In another example, the D-bound entropy for the various possible black hole solutions on a four-dimensional brane have been considered in [39] . It is found that the D-bound entropy for this solution is apparently different from that obtained for the four-dimensional black hole solutions. This difference is considered as an extra loss of information which comes from the extra dimension, when an extra-dimensional black hole is moved out of the observer's cosmological horizon. The obtained results there also have been considered, by adopting the recent Bohr-like approach to black hole quantum physics for excited black holes [39].

\section{Surrounded Vaidya black hole solution}

The metric of Vaidya black hole solution surrounded by cosmological fields introduced in $[28,40]$ is given by

$$
\begin{aligned}
\mathrm{d} s^{2}= & -\left(1-\frac{2 M(u)}{r}-\frac{N_{s}(u)}{r^{3 \omega_{s}+1}}\right) \mathrm{d} u^{2} \\
& +2 \epsilon \mathrm{d} u \mathrm{~d} r+r^{2} \mathrm{~d} \Omega^{2}
\end{aligned}
$$

where $M(u), N_{s}(u)$ and $\omega_{s}$ are the black hole dynamical mass, the surrounding field's characteristic parameter and the equation of state parameter of the surrounding field, respectively. As mentioned in [40], the metric (6) is a solution to the Einstein field equations in four dimension for a fluid that is not a perfect fluid in general. However, the "averaged" energy-momentum defined as $T_{v}^{\mu}=\left(-\rho,\left\langle\mathcal{T}_{j}^{i}\right\rangle\right)$ where $\left\langle\mathcal{T}_{j}^{i}\right\rangle=\frac{\alpha}{3} \rho_{s}(u, r) \delta_{j}^{i}=p_{s}(u, r) \delta_{j}^{i}$, can be treated as an effective perfect fluid.

In contrast to stationary spacetimes, the local definitions of the various horizons do not necessarily coincide with the location of the event horizon for dynamical black holes [41]. For such dynamical spacetimes, one is left with the question: "For which surface should one define the black hole area, surface gravity, temperature or entropy?". The canonical choice is to use the event horizon. However, there is some evidence that it is the apparent horizon, and not the event horizon, that plays the key role in the Hawking radiation [42-45]; see also [46-48]. This finding has become a key point in the hope to demonstrate the Hawking radiation in the laboratory using models of analogue gravity [49]. Therefore, we consider the BekensteinHawking entropy for apparent horizons associated to the metric (6) with various cosmological fields. Then we derive 
the D-bound and the Bekenstein bound for these backgrounds.

In the following sections, we investigate the D-bound and the Bekenstein bound for a Vaidya black hole surrounded by various cosmological fields. Since the matter source supporting the geometry (6) is not a perfect fluid [28,40], we call these fields quintessence-like, phantom-like and cosmological constant-like fields, since they possess an average equation of state like the quintessence, phantom and cosmological constant in the standard model of cosmology. Then we compare the D-bound with the Bekenstein bound to show that the more cosmological fields are diluted, the more the D-bound and the Bekenstein bound are identified. The Bekenstein bound is an entropy bound over a matter system which is saturated for black holes. This bound is the direct result of GSL. On the other hand, the D-bound is also is a direct result of GSL which in our model puts an upper bound on the entropy of the matter system. This upper bound cannot be greater than the Bekenstein bound. Also it should not be less than the saturated form of the Bekenstein bound because a black hole possesses a certain entropy. Hence, one can admit the identification of these two bounds at least for black holes as the local matter systems.

Here, we deem it necessary to explain a delicate point. The D-bound entropy is essentially defined for a local matter system before and after its local-crossing through the cosmological apparent horizon of an observer [22]. But here we have considered a local matter system surrounded by a global cosmological field (quintessence or phantom), and we applied the D-bound on this combined "local-global" system, the global part of which has no local behavior at all in crossing the cosmological apparent horizon of the observer. How can this discrepancy be solved? To properly address this point, we may proceed as follows. The direct application of the D-bound is meaningless for the cosmological fields because no observer can imagine a "global" cosmological field crossing "locally" through his/her cosmological apparent horizon. However, for a combined system of local matter and global cosmological fields one may still apply the D-bound by ignoring the direct roles of global cosmological fields in the D-bound scenario and merely resorting to their indirect roles on the local behaviors of the matter. For a local black hole, surrounded by cosmological fields, these indirect (effective) impacts can be a shift in the locus of black hole's horizon and the appearance of new horizons. Therefore, the comparison of the Dbound and the Bekenstein bound for these combined systems is established through considering merely the local behaviors of the modified horizons, and the identification of these bounds leads to ruling out the combined system of a black hole surrounded by a quintessence or phantom field.

\section{D-bound and Bekenstein bound for surrounded Vaidya solution by cosmological constant-like field}

\subsection{D-bound}

Considering the equation of state parameter by $\omega_{c}=-1$ $[28,40]$, the metric (6) becomes

$$
\begin{aligned}
\mathrm{d} s^{2}= & -\left(1-\frac{2 M(u)}{r}-N_{c}(u) r^{2}\right) \mathrm{d} u^{2} \\
& +2 \mathrm{~d} u \mathrm{~d} r+r^{2} \mathrm{~d} \Omega_{2}^{2},
\end{aligned}
$$

where $N_{c}(u)$ is the normalization parameter for the cosmological field surrounding the black hole. This metric describes a black hole surrounded by a cosmological constant-like field. The positive energy condition on the surrounding cosmological field leads to $N_{c}(u)>0$ [28]. The cosmological background which has negative surface gravity decreases the gravitational attraction of the black hole. This repulsive gravitational effect with the equation of state parameter $\omega_{c}=-1$ makes the cosmological constant field the most favored candidates for the dark energy responsible for the accelerating expansion of the universe [50]. The metric (7) indicates the non-trivial effects of the surrounding cosmological field which differs from the Vaidya black hole in an empty background. The background cosmological field changes the causal structure of the Vaidya black hole in an empty space. The causal structure change of Vaidya to Vaidyade Sitter space is similar to the causal structure change of Schwarzschild to Schwarzschild-de Sitter space [51].

To derive the D-bound for the Vaidya case one needs the apparent cosmological horizon, which will be described completely in this section. First, we have to find the horizons of this solution. In Ref. [28], the black hole horizon and the apparent cosmological horizon are obtained for the Vaidya solution surrounded by a cosmological constant-like field in detail. There are black hole and apparent cosmological horizons, subject to the particular condition $\Delta(u)=$ $1-27 M^{2}(u) N_{c}(u)>0$, representing the inner and outer horizons, respectively as [28]

$$
\begin{aligned}
r_{A H^{-}}= & 2 M(u)+8 M^{3}(u) N_{c}(u)+O\left(N_{c}^{2}(u)\right), \\
r_{A H^{+}}= & \frac{1}{\sqrt{N_{c}(u)}}-M(u) \\
& -\frac{3}{2} M^{2}(u) \sqrt{N_{c}(u)}-4 M^{3}(u)+O\left(N_{c}^{\frac{3}{2}}(u)\right) .
\end{aligned}
$$

The inner apparent horizon $r_{A H^{-}}$is larger than the dynamical Schwarzschild radius $r(u)=2 M(u)$ and the outer cosmological apparent horizon $r_{c}=r_{A H^{+}}$tends to infinity for $N_{c}(u) \lll 1$. The cosmological field and the black hole mass have positive contributions to the inner apparent horizon, whereas the black hole mass has a negative contribution to the outer horizon and pulls the cosmological horizon back 
towards the center of the black hole. The black hole evaporation leads to shrinking and vanishing of the inner apparent horizon, while the outer horizon is tending to its asymptotic value $N_{c}^{-\frac{1}{2}}$.

Now, we apply Bousso's method like the one defined to some extent in Sect. 2. We consider an observer inside a system which is circumscribed by a sphere of radius $r_{A H^{+}}$ (9). Then we assume that the observer moves away from the matter system (black hole) until he/she observes that the matter system crosses out his cosmological apparent horizon with radius of $r_{A H^{+}}$. GSL claims that the entropy of the final state of this apparent cosmological horizon in the absence of a black hole is greater than the entropy of the initial state of this apparent cosmological horizon with a black hole. The final state system circumscribing by a sphere with the radius $r_{0}$ has entropy $S_{0}=\frac{A_{0}}{4}=\pi r_{0}^{2}$, where $A_{0}$ is the area of the apparent cosmological horizon in the absence of the matter system $(M(u)=0)$ and $r_{0}=r_{c}(M(u)=0)=N_{c}^{-\frac{1}{2}}$. The entropy of the initial state system is the sum of the matter system (black hole) entropy $S_{m}=S_{A H^{-}}$and the entropy of the cosmological horizon $S_{\mathrm{AH}^{+}}$. We can write them as follows:

$$
\begin{aligned}
S_{A H^{-}}= & \pi r_{A H^{-}}^{2} \\
= & \left.\pi\left(4 M^{2}(u)+32 M^{4}(u) N_{c}(u)\right)+O\left(N_{c}^{2}(u)\right)\right), \quad(10) \\
S_{A H^{+}}= & \pi r_{A H^{+}}^{2} \\
= & \pi\left(\frac{1}{N_{c}(u)}-2 \frac{M(u)}{\sqrt{N_{c}}}-2 M^{2}(u)\right. \\
& \left.-5 M^{3}(u) \sqrt{N_{c}(u)}-16 M^{4}(u) N_{c}(u)\right)+O\left(N_{c}^{\frac{3}{2}}(u)\right)
\end{aligned}
$$

According to GSL the final entropy $S_{0}=\frac{A_{0}}{4}$ is greater than initial entropy $S_{A H^{-}}+S_{A H^{+}}$. Thus, using (10) and (11) in $S_{0} \geqslant S_{A H^{-}}+S_{A H^{+}}$, we obtain

$$
\begin{aligned}
S_{m} \leqslant & \pi\left(2 \frac{M(u)}{\sqrt{N_{c}}}+2 M^{2}(u)\right. \\
& \left.\left.+5 M^{3}(u) \sqrt{N_{c}(u)}+16 M^{4}(u) N_{c}(u)\right)\right) .
\end{aligned}
$$

This is the D-bound for the Vaidya solution with surrounding by a cosmological constant-like field. When the gravitational radius of the matter system $r_{g}$ is much smaller than the cosmological radius $r_{c}$, the system is called "dilute", i.e. when $r_{g} \ll r_{c}$. In the Vaidya solution it means that $N_{c}(u) \lll 1$, leading to a large radius for the cosmological horizon. In the dilute limit, i.e. $N_{c}(u) \lll 1$, the inequality (12) becomes

$$
S_{m} \leqslant 2 \pi \frac{M(u)}{\sqrt{N_{c}}} .
$$

The inequality (13) puts an upper bound for the entropy of the black hole. The normalization parameter for the cosmological field $N_{c}(u)$ in the limit of a dilute field makes larger the upper bound for the black hole entropy but the black hole mass $M(u)$ has an opposite role. One can recognize from inequality (12) that all terms in RHS are positive or both parameters $M(u)$ and $N(u)$ have positive effects on the upper entropy of black hole, imposed by the D-bound.

\subsection{Bekenstein bound}

To derive the Bekenstein bound (1) for the Vaidya solution with surrounding by a cosmological constant-like field we need to know the radius of the sphere $R$ circumscribing the system and its energy $E$. To find the Bekenstein bound we will apply Bousso's method [22]. For the Vaidya black hole surrounded by a cosmological field, the energy of the system is not well defined, due to the lack of a suitable asymptotic region. However, there exists a solution which is known as the Vaidya black hole solution surrounded by a cosmological field which behaves like the metric of a de Sitter space with cosmological horizon radius $r_{c}$, at large distances. This solution is like the "system's equivalent black hole", and its radius is like the "system's gravitational radius" $r_{g}$. The $r_{g}$ for the Schwarzschild black hole equals twice the energy of the black hole, which is the same as the event horizon radius of the black hole. But for this solution there are some delicate points, as follows. Here $r_{g}$ is the same as the apparent horizon of the black hole, but it is not the same as twice the energy of the black hole. Thus, the corrected $r_{g}$ and cosmological horizon $r_{c}$ are

$$
\begin{aligned}
r_{g}= & 2 m=r_{A H^{-}} \\
= & 2 M(u)+8 M^{3}(u) N_{c}(u)+O\left(N_{c}^{2}(u)\right), \\
r_{c}= & r_{A H^{+}}=\frac{1}{\sqrt{N_{c}(u)}}-M(u) \\
& -\frac{3}{2} M^{2}(u) \sqrt{N_{c}(u)}-4 M^{3}(u)+O\left(N_{c}^{\frac{3}{2}}(u)\right) .
\end{aligned}
$$

The Bekenstein bound for the system's equivalent black hole with gravitational radius $r_{g}$ is written as follows [22]:

$S_{m} \leqslant \pi r_{g} R$,

where $R$ is radius of the sphere which circumscribes the system. Here, $R$ is equal to $r_{c}$. Now, we put Eqs. (14) and (15) into (16). Then we have

$$
\begin{aligned}
S_{m} \leqslant & \pi\left(\frac{2 M(u)}{\sqrt{N_{c}(u)}}+5 M^{3}(u) \sqrt{N_{c}(u)}\right. \\
& -2 M^{2}(u)-8 M^{4}(u)-8 M^{4}(u) N_{c}(u) \\
& -32 M^{6} N_{c}(u)+O\left(N_{c}^{\frac{3}{2}}(u)\right) .
\end{aligned}
$$


We see that in the inequality (17) for $N_{c}(u) \lll 1$ the first term dominates which leads exactly to the D-bound. However, in inequality (12) for $N_{c}(u) \lll 1$ the dominant term is the first term which is exactly the same as the dominant term in Eq. (17) (i.e. $S_{m} \leqslant \pi \frac{2 M(u)}{\sqrt{N_{c}(u)}}$ ). So, the Bekenstein bound and the D-bound (13) are identified for very diluted surrounding field. For the case of a little less diluted surrounding field, i.e. $N_{c}(u) \ll 1$, the Bekenstein bound (17) reads

$S_{m} \leqslant \pi\left(\frac{2 M(u)}{\sqrt{N_{c}(u)}}-2 M^{2}(u)-8 M^{4}(u)\right)$,

which is a tighter bound than the D-bound $S_{m} \leqslant \pi\left(\frac{2 M(u)}{\sqrt{N_{c}(u)}}+\right.$ $2 M^{2}(u)$ ) derived by this less dilute approximation from (12).

When the field is strong and the system is not dilute, the bounds (12) and (17) are not identified. Except for a very dilute system limit, the Bekenstein bound (17) for the Vaidya solution with surrounding by a cosmological constant-like field and its D-bound (12) are not the same. The parameters $N_{c}(u)$ and $M(u)$ always have positive effects in the D-bound, but in the Bekenstein bound they have both positive and negative contributions. If negative parts dominate in the Bekenstein bound over positive ones, then the RHS in (18) becomes negative, which is physically meaningless. The requirement of a positive upper bound in the Bekenstein bound sets a constraint on the parameters $N_{c}(u)$ and $M(u)$ in (18). There is no such constraint for the D-bound (12) regarding this solution because the RHS in (12) is always positive.

\section{D-bound and the Bekenstein bound for the Vaidya black hole with surrounding quintessence-like field}

\subsection{D-bound}

Considering the equation of state parameter $\omega_{q}=-\frac{2}{3}[28$, 40], the metric (6) becomes

$\mathrm{d} s^{2}=-\left(1-\frac{2 M(u)}{r}-N_{q}(u) r\right) \mathrm{d} u^{2}+2 \mathrm{~d} u \mathrm{~d} r+r^{2} \mathrm{~d} \Omega_{2}^{2}$.

This metric describes a black hole surrounded by a quintessencelike field. Here, $N_{q}(u)$ is the normalization parameter for the quintessence-like field surrounding the black hole. The positive energy condition on the surrounding quintessence-like field leads to $N_{q}>0$ [28]. According to the metric (19), it is obvious that the surrounding quintessence-like field has a non-trivial contribution to the metric of the Vaidya black hole. The background quintessence-like field changes the causal structure of the black hole solution in comparison to that of the original Vaidya black hole in an empty background. An almost similar effect occurs when one immerses a Schwarzschild black hole in a background which is asymptotically de Sitter [51]. Similar to a Vaidya black hole surrounded by a cosmological field, the surface gravity of the black hole here is also negative and it leads to gravitational repulsion.

Deriving the D-bound for this case is the same as the one which we derived for Vaidya black hole solution surrounded by a cosmological field in the previous section. For $\Delta(u)=1-8 M(u) N_{q}(u)>0$, there are two physical inner and outer apparent horizons [28]. The locations of the two apparent horizons for $\Delta(u)>0$ with small quintessence normalization parameters $\left(N_{q} \ll M(u)\right)$ are

$$
\begin{aligned}
r_{A H^{-}}= & 2 M(u)+4 M^{2}(u) N_{q}(u)+O\left(N_{q}^{2}(u)\right), \\
r_{A H^{+}}= & \frac{1}{N_{q}(u)}-2 M(u) \\
& -4 M^{2}(u) N_{q}(u)+O\left(N_{q}^{2}(u)\right) .
\end{aligned}
$$

The surrounding quintessence field has contributions both in the physical inner horizon $r_{A H^{-}}$(which is larger than the dynamical Schwarzschild radius $r(u)=2 M(u)$ ) and the outer horizon $r_{A H^{+}}$(cosmological horizon) tending to infinity for $N_{q}(u) \lll 1$. The quintessence field and the black hole mass have positive contributions for the inner apparent horizon. The black hole mass has a negative contribution for the outer horizon and it pulls the cosmological horizon toward inside. The black hole evaporation leads to shrinking and vanishing of the inner apparent horizon, while the outer horizon is tending to its asymptotic value $N_{q}^{-1}$.

The existence of two apparent horizons is guaranteed considering the D-bound. Here, the entropy of the final state system is $S_{0}=\frac{A_{0}}{4}$, where $A_{0}$ is the area of the cosmological horizon within which there is no matter system except for the quintessence field. The initial state entropy is the sum of the black hole entropy $S_{m}=S_{A H^{-}}$and the cosmological horizon entropy $S_{A H^{+}}$. They are given by

$$
\begin{aligned}
S_{A H^{-}}= & \pi r_{A H^{-}}^{2} \\
= & \pi\left(4 M^{2}(u)+16 M^{3}(u) N_{q}(u)\right)+O\left(N_{q}^{2}(u)\right) \\
S_{A H^{+}}= & \pi r_{A H^{+}}^{2}=\pi\left(\frac{1}{N_{q}^{2}(u)}-\frac{4 M(u)}{N_{q}(u)}\right. \\
& \left.-4 M^{2}(u)-16 M^{3}(u) N_{q}(u)\right)+O\left(N_{q}^{2}(u)\right) .
\end{aligned}
$$

According to GSL the final entropy $S_{0}=\frac{A_{0}}{4}$ is greater than the initial entropy $S_{A H^{-}}+S_{A H^{+}}$. Thus, by using (22), (23) and $r_{0}=r_{c}(M(u)=0)=N_{q}^{-1}(u)$ in $S_{0} \geqslant S_{A H^{-}}+S_{A H^{+}}$ we have

$S_{m} \leqslant \pi\left(\frac{4 M(u)}{N_{q}(u)}+4 M^{2}(u)+16 M^{3}(u) N_{q}(u)\right)$. 
In the limit of $N_{q}(u) \lll 1$ the above inequality becomes

$S_{m} \leqslant \pi \frac{4 M(u)}{N_{q}(u)}$.

The inequality (25) puts an upper bound for the entropy of the black hole. As the black hole mass increases or the background field dilutes $N_{q}(u) \lll 1$, the bound becomes looser.

\subsection{Bekenstein bound}

In this case, the derivation method of the Bekenstein bound (1) is the same as the method we used in the previous section for the Vaidya solution surrounded by a cosmological field. Regarding inequality (16), the radius $R$ of the sphere circumscribing the system and gravitational radius $r_{g}$ are necessary for considering the Bekenstein bound. The gravitational radius $r_{g}$ here is not twice the energy of the Schwarzschild black hole. It is not well defined, because of the lack of an asymptotic flat region, for a Vaidya black hole surrounded by a quintessence field. However, in this solution $r_{g}$ is the location of the apparent horizon of the black hole $r_{A H^{-}}$. Also the radius $R$ in this solution is equal to the cosmological apparent horizon $r_{C}=r_{A H^{+}}$. They are given as follows:

$r_{g}=2 m=r_{A H^{-}}=2 M(u)+4 M^{2}(u) N_{q}(u)+O\left(N_{q}^{2}(u)\right)$,

$$
\begin{aligned}
r_{c} & =r_{A H^{+}} \\
& =\frac{1}{N_{q}(u)}-2 M(u)-4 M^{2}(u) N_{q}(u)+O\left(N_{q}^{2}(u)\right) .
\end{aligned}
$$

Now, one can put Eqs. (26) and (27) into Eq. (16) to derive the Bekenstein bound:

$S_{m} \leqslant \pi\left(\frac{2 M(u)}{N_{q}(u)}-16 M^{3}(u) N_{q}(u)\right)+O\left(N_{q}^{2}(u)\right)$.

Similar to the previous case, we use $R=r_{c}$ as the radius of the sphere which circumscribes the system. In the Bekenstein bound (28), in the limit of very dilute energy $N_{q}(u) \lll$ 1 , the dominant term is the first term (i.e. $S_{m} \leqslant \pi \frac{2 M(u)}{N_{q}(u)}$ ) which is the same Bekenstein bound as for the Vaidya black hole surrounded by a quintessence field, and in this limit of very dilute energy, namely $N_{q}(u) \lll 1$, the Bekenstein bound (28) is twice tighter than the D-bound (25). Therefore, for quintessence background the D-bound does not give the Bekenstein bound.

In this limit, the normalization parameter $N_{q}(u)$, for $N_{q}(u) \lll 1$ makes the upper Bekenstein bound larger and the mass of the black hole does the same job for large amounts of mass. As $N_{q}(u)$ increases, the absolute values of the first and the second terms decrease and increase, respectively, in the RHS of (28) and make the bound tighter. Overall, it turns out that the Bekenstein bound here is tighter than the Dbound. In the D-bound the mass of the black hole has always a positive contribution on the upper bound, but in the Bekenstein bound (28) the mass of the black hole may have both positive and negative contributions.

\section{D-bound and the Bekenstein bound for Vaidya black hole surrounded by phantom-like field}

\subsection{D-bound}

Considering the equation of state parameter $\omega_{p}=-\frac{4}{3}[28$, 40], the metric (6) becomes

$\mathrm{d} s^{2}=-\left(1-\frac{2 M(u)}{r}-N_{p}(u) r^{3}\right) \mathrm{d} u^{2}+2 \mathrm{~d} u \mathrm{~d} r+r^{2} \mathrm{~d} \Omega_{2}^{2}$,

which describes a black hole embedded in a phantom background. Here, $N_{p}(u)$ is the normalization parameter for the phantom-like field surrounding the black hole. The positive energy condition on the surrounding phantom field leads to $N_{p}>0$ [28]. According to the metric (29), the surrounding phantom field has a non-trivial effect on the Vaidya black hole and its causal structure. In this case, like the previous cases, the phantom-like background field causes a negative surface gravity, which leads to gravitational repulsion [50].

In deriving the D-bound for this case, we are interested in the solutions with two apparent horizons, one of them is the black hole's apparent horizon and the other one plays the role of the cosmological horizon $r_{c}$. These solutions are obtained by the condition $\Delta(u)=1-\frac{2048}{27} M^{3}(u) N_{p}(u)>0$ as [28]. They are as follows:

$$
\begin{aligned}
r_{A H^{-}}= & 2 M(u)+16 M^{4}(u) N_{p}(u)+O\left(N_{p}^{2}(u)\right), \\
r_{A H^{+}}= & \frac{1}{N_{p}^{\frac{1}{3}}(u)}-\frac{2}{3} M(u) \\
& -\frac{8}{9} M^{2}(u) N_{p}^{\frac{1}{3}}(u)-\frac{160}{81} M^{3}(u) N_{p}^{\frac{2}{3}}(u) \\
& -\frac{16}{3} M^{4}(u) N_{p}(u)+O\left(N_{p}^{\frac{4}{3}}(u)\right),
\end{aligned}
$$

where $r_{A H^{-}}$and $r_{A H^{+}}$represent the inner and outer physical apparent horizons, respectively. Thus, the black hole in a phantom background possesses an inner horizon larger than the dynamical Schwarzschild radius (apparent horizon) $r(u)=2 M(u)$ and an outer horizon, which is the cosmological apparent horizon, blows up for $N_{p} \lll 1$. Regarding Eqs. (30) and (31), one can realize that the phantom field makes the inner apparent horizon larger and the black hole mass makes the outer horizon larger. The black hole evaporation process shrinks the inner apparent horizon, while the outer one closes to its asymptotic value $N_{p}^{-1 / 3}$. 
The existence of two apparent horizons is guaranteed considering the D-bound. Here, the entropy of the final state is $S_{0}=\frac{A_{0}}{4}$, where $A_{0}$ is the area of the cosmological horizon in the absence of a matter system except for the phantom field. The initial state entropy is the sum of the black hole entropy $S_{m}=S_{A H^{-}}$and the cosmological horizon entropy $S_{A H^{+}}$given as

$$
\begin{aligned}
S_{A H^{-}}= & \pi r_{A H^{-}}^{2} \\
= & \pi\left(4 M^{2}(u)+64 M^{5}(u) N_{p}(u)\right)+O\left(N_{p}^{2}(u)\right), \\
S_{A H^{+}}= & \pi r_{A H^{+}}^{2} \\
= & \pi\left(\frac{1}{N_{p}^{\frac{2}{3}}(u)}-\frac{4}{3} \frac{M(u)}{N_{p}^{\frac{1}{3}}(u)}-\frac{4}{3} M^{2}(u)\right. \\
& -\frac{224}{81} M^{3}(u) N_{p}^{\frac{1}{3}}(u)-\frac{1760}{243} M^{4}(u) N_{p}^{\frac{2}{3}}(u) \\
& \left.-\frac{64}{3} M^{5}(u) N_{p}(u)\right)+O\left(N_{p}^{\frac{4}{3}}(u)\right) .
\end{aligned}
$$

According to GLS and by using (32), (33) and $r_{0}=$ $r_{c}(M(u)=0)=N_{p}^{-\frac{1}{3}}$ in $S_{0} \geqslant S_{A H^{-}}+S_{A H^{+}}$, one can derive the D-bound for this solution as

$$
\begin{aligned}
S_{m} \leqslant & \pi\left(\frac{4}{3} \frac{M(u)}{N_{p}^{\frac{1}{3}}(u)}+\frac{4}{3} M^{2}(u)+\frac{224}{81} M^{3}(u) N_{p}^{\frac{1}{3}}(u)\right. \\
& \left.+\frac{1760}{243} M^{4}(u) N_{p}^{\frac{2}{3}}(u)+\frac{64}{3} M^{5}(u) N_{p}(u)\right) .
\end{aligned}
$$

In the limit $N_{p} \lll 1$, the D-bound (34) becomes

$$
S_{m} \leqslant \frac{4}{3} \pi \frac{M(u)}{N_{p}^{\frac{1}{3}}(u)} .
$$

The inequality (35) represents an upper bound for the entropy of the black hole. The upper bound entropy becomes larger for a large black hole mass $M(u)$ and a small normalization parameter $N_{p}(u)$.

\subsection{Bekenstein bound}

The gravitational radius and the outer cosmological apparent horizon in this case are obtained:

$$
\begin{aligned}
r_{g}= & 2 m=r_{A H^{-}} \\
= & 2 M(u)+16 M^{4}(u) N_{p}(u)+O\left(N_{p}^{2}(u)\right), \\
r_{c}= & r_{A H^{+}}=\frac{1}{N_{p}^{\frac{1}{3}}(u)}-\frac{2}{3} M(u) \\
& -\frac{8}{9} M^{2}(u) N_{p}^{\frac{1}{3}}(u)-\frac{160}{81} M^{3}(u) N_{p}^{\frac{2}{3}}(u) \\
& -\frac{16}{3} M^{4}(u) N_{p}(u)+O\left(N_{p}^{\frac{4}{3}}(u)\right) .
\end{aligned}
$$

We can put Eqs. (36) and (37) into Eq. (16) to derive the Bekenstein bound for $N_{p} \lll 1$ :

$S_{m} \leqslant \pi\left(\frac{2 M(u)}{N_{p}^{\frac{1}{3}}(u)}-\frac{4}{3} M^{2}(u)\right)$.

In the Bekenstein bound (16) we put $R=r_{c}$ as the radius of the sphere circumscribing the system. In this case, if $\frac{1}{N_{p}^{\frac{1}{3}}(u)}<4 M(u)$ the Bekenstein bound (38) will be tighter than the D-bound (35). But we know that $\Delta(u)=1-$ $\frac{2048}{27} M^{3}(u) N_{p}(u)>0$, which leads to $\frac{1}{N_{p}^{\frac{1}{3}}(u)}>4 M(u)$ giving two real solutions as the physical apparent horizons which are necessary for considering the D-bound. The other amounts of $\Delta$ (i.e $\Delta(u) \leqslant 0$ ) which lead to $\frac{1}{N_{p}^{\frac{1}{3}}(u)}<4 M(u)$ cannot give two physical apparent horizons as solutions. So, the Bekenstein bound here cannot be tighter than the Dbound. Therefore, if $\frac{1}{N_{p}^{\frac{1}{3}}(u)}>4 M(u)$ the D-bound will be tighter than the Bekenstein bound. However, for $\frac{1}{N_{p}^{\frac{1}{3}}(u)}=$ $4 M(u)$ there is no D-bound because we do not have two physical apparent horizons.

\section{Conclusions and results}

The D-bound entropy is essentially defined for a matter system which crossing a cosmological apparent horizon of the observer who is near the matter system. Hence the definition of the D-bound is based on the existence of a cosmological horizon far from the local matter system and their corresponding entropies. Since the cosmological field fills the whole of the system and defines the cosmological horizon, the global structure of the system is important. In principle, we use each of the D-bound and the Bekenstein bound to find a constraint on the entropy of the matter system. The constraint on the cosmological field surrounding the matter system is a consequence of the "identification" of these two bounds. This demand for the identification of these two bounds is based on the GSL. We have derived the D-bound for the Vaidya solutions surrounded by cosmological fields and indicated that for the one particular solution the D-bound is the same as the Bekenstein bound in dilute systems. Before presenting our conclusions and results for the D-bound and the Bekenstein bound defined for the matter system's equivalent black hole, it is worth to mention that the Vaidya geometry studied in this work also admits naked singularity type solutions [63]. The possibility of the formation of naked singularities for the solution (6) is addressed in [40]. In summary, if the discriminant (Eq. (25) in [40]) for the null geodesics admits 
a positive real root, then the solution describes a naked singularity and consequently provides a counterexample for the Penrose cosmic censorship conjecture [23]. In contrast to black holes, naked singularities do not have any apparent or event horizon, and then one cannot adjust a suitable radius circumscribing these systems. As a consequence, it makes it difficult to define a Bekenstein bound for such systems. The same argument applies to the case of the D-bound that is defined based on the existence of a cosmological horizon and a matter system which is called by Bousso the system's equivalent black hole, possessing a radius named the system's gravitational radius, $r_{g}$ [22]. For the case of a naked singularity, the definition of a suitable gravitational radius $r_{g}$ is not trivial.

The results obtained in the present work are as follows:

- The D-bound for a Vaidya solution surrounded by a cosmological constant-like field is the same as the Bekenstein bound in the dilute system limit. As the background field becomes more considerable, the equality of the Dbound and the Bekenstein bound is more hampered. In the case of a dilute cosmological constant-like field, the contribution of the background field in the metric is $N_{c} r^{2}$ which leads to the equality of the D-bound with the Bekenstein bound.

- The D-bound for the Vaidya black hole surrounded by an exotic quintessence-like field is the same as the Bekenstein bound in a light background field, except for a constant coefficient 2. Since the contribution of background field in the metric is $N_{q} r$, which is weaker than the case of a cosmological constant-like field $N_{c} r^{2}$ at $r>1$, the D-bound does not again coincide with the Bekenstein bound, even in light background systems. In a light quintessence-like background field the D-bound is looser than the Bekenstein bound.

- The D-bound for the Vaidya black hole surrounded by an exotic phantom-like field is not the same as the Bekenstein bound in a dilute phantom background field. Since the contribution of the background field in the metric is $N_{p} r^{3}$, which is stronger than the case of cosmological constant-like field $N_{c} r^{2}$ at $r>1$, the D-bound does not again coincide with the Bekenstein bound, even in light background systems. The D-bound is tighter than the Bekenstein bound for the light phantom background.

The conclusions are as follows. The dynamical background fields, possessing cosmological horizons, play the role of a repulsion force like the case of a cosmological constant which manifests itself in the metric as $r^{2}$. For this repulsion force, the D-bound is identified with the Bekenstein bound in dilute systems. Any deviation from the $r^{2}$ term corresponding to the quintessence and phantom fields with contributions as $r$ and $r^{3}$ terms, having less and more repulsion forces than that of the cosmological constant, leads to D-bounds looser and tighter than the Bekenstein bound, respectively. Finally, it is worth mentioning that the D-bound and the Bekenstein bound are direct consequences of GSL. Therefore, we conclude that they should lead to the same entropy bound imposed on a certain matter system. This conclusion leads to one possible option as follows:

- Cosmological constant-like field viability: The cosmological constant-like field has a reasonable behavior, to be compared with two other cosmological fields, namely the quintessence and phantom fields, regarding the identification of the D-bound and the Bekenstein bound for light systems. It seems that by implementation of a thermodynamical criterion, namely the identification of the D-bound and the Bekenstein bound, on the Vaidya black hole solution surrounded by cosmological fields, one may exclude the quintessence and phantom fields and just keep the cosmological constant as the single field for which the D-bound and the Bekenstein bound are exactly identified. $^{2}$

We have studied the same thermodynamical criterion on the other known dynamical black hole solutions surrounded by cosmological fields and observed that the cosmological constant is preferred as the viable cosmological field rather than the other known cosmological fields [61,62]. In [62], the hypothesis of the "D-bound-Bekenstein bound identification" has been applied for the McVittie solution surrounded by cosmological fields in the dilute limit and it has been indicated that this criterion is only true for the cosmological constant field as a candidate for the dark energy, and the other cosmological fields, such as phantom and quintessence fields, do not satisfy this criterion. Also in [61] the same criterion is applied for the universe system and the universe-black hole system, which is dominated by quintessence, phantom or cosmological constant fields. By entropic considerations, it turns out that, for both systems, the cosmological constant field is the only viable field rather than the others.

Data Availability Statement This manuscript has no associated data or the data will not be deposited. [Authors' comment: This manuscript presents only theoretical and mathematical results.]

Open Access This article is licensed under a Creative Commons Attribution 4.0 International License, which permits use, sharing, adaptation, distribution and reproduction in any medium or format, as long as you give appropriate credit to the original author(s) and the source, provide a link to the Creative Commons licence, and indicate if changes were made. The images or other third party material in this article are included in the article's Creative Commons licence, unless indicated otherwise in a credit line to the material. If material is not

\footnotetext{
2 The violation of the second law of thermodynamics by quintessence and phantom fields which represents their unphysical behaviors in many ways, has been discussed in [53-59].
} 
included in the article's Creative Commons licence and your intended use is not permitted by statutory regulation or exceeds the permitted use, you will need to obtain permission directly from the copyright holder. To view a copy of this licence, visit http://creativecomm ons.org/licenses/by/4.0/.

Funded by SCOAP ${ }^{3}$.

\section{References}

1. P.J. Steinhardt, in Critical Problems in Physics, ed. by V.L. Fitch, R. Marlow (Princeton, Princeton University Press, 1997)

2. I. Zlatev, L.M. Wang, P.J. Steinhardt, Phys. Rev. Lett. 82, 896 (1999)

3. P.J. Steinhardt, L.M. Wang, I. Zlatev, Phys. Rev. D 59, 123504 (1999)

4. S. Weinberg, Rev. Mod. Phys. 61, 1 (1989)

5. R.R. Caldwell, R. Dave, P.J. Steinhardt, Phys. Rev. Lett. 80, 1582 (1998)

6. Y. Fujii, Phys. Rev. D 26, 2580 (1982)

7. L.H. Ford, Phys. Rev. D 35, 2339 (1987)

8. C. Wetterich, Nucl. Phys. B 302, 668 (1988)

9. B. Ratra, P.J.E. Peebles, Phys. Rev. D 37, 3406 (1988)

10. S.M. Carroll, M. Hoffman, M. Trodden, Phys. Rev. D 68, 023509 (2003)

11. P. Singh, M. Sami, N. Dadhich, Phys. Rev. D 68, 023522 (2003)

12. M. Sami, A. Toporensky, Mod. Phys. Lett. A 19, 1509 (2004)

13. J.D. Bekenstein, Phys. Rev. D 7, 2333 (1973)

14. J.D. Bekenstein, Lett. Nuovo Cim. 11, 467 (1974)

15. S.W. Hawking, Commun. Math. Phys. 43, 199 (1975)

16. C. Corda, Class. Quantum Gravity 32, 195007 (2015)

17. J.D. Bekenstein, Phys. Rev. D 23, 287 (1981)

18. H.J. Bremermann, Optimization through evolution and recombination, in Self-Organizing Systems 1962, ed. by M.C. Yovitts, et al. (Spartan Books, Washington, DC, 1962), pp. 93-106

19. T. Jacbson, Phys. Rev. Lett. 75, 1260 (1995)

20. J.D. Bekenstein, Cont. Phys. 45(1), 31 (2005)

21. R. Bousso, JHEP 07, 004 (1999)

22. R. Bousso, JHEP 0104, 035 (2001)

23. R. Penrose, Riv. Nuovo Cimento 1, 252 (1969)

24. S.G. Ghosh, N. Dadhich, Phys. Rev. D 64, 047501 (2001)

25. I.H. Dwivedi, P.S. Joshi, Class. Quantum Gravity 6(11), 1599 (1989)

26. Y. Kuroda, Y. Prog, Theor. Phys. 72(1), 63 (1984)
27. T. Harko, Phys. Rev. D 68, 064005 (2003)

28. Y. Heydarzade, F. Darabi, Eur. Phys. J. C 78, 342 (2018)

29. J.D. Bekenstein, Phys. Rev. D 30, 1669 (1984)

30. M. Schiffer, J.D. Bekenstein, Phys. Rev. D 39, 1109 (1989)

31. D.N. Page, Subsystem entropy exceeding Bekenstein's bound. arXiv:hep-th/0007237 (2000)

32. J.D. Bekenstein, On Page's examples challenging the entropy bound. arXiv:gr-qc/0006003 (2000)

33. R.M. Wald, Living Rev. Relativ. 4, 6 (2001)

34. J.D. Bekenstein, Nuovo Cim. Lett. 4, 737 (1972)

35. J.D. Bekenstein, Phys. Rev. D 9, 3292 (1974)

36. W.G. Unruh, R.M. Wald, Phys. Rev. D 25, 942 (1982)

37. W.G. Unruh, R.M. Wald, Phys. Rev. D 27, 2271 (1983)

38. M.A. Pelath, R.M. Wald, Phys. Rev. D 60, 104009 (1999)

39. Y. Heydarzade, H. Hadi, C. Corda, F. Darabi, Phys. Lett. B 776, 457 (2018)

40. Y. Heydarzade, F. Darabi, Eur. Phys. J. C 78, 582 (2018)

41. A.B. Nielsen, Black holes as local horizons. arXiv:0711.0313

42. P. Hajicek, Phys. Rev. D 36, 1065 (1987)

43. W.A. Hiscock, Phys. Rev. D 40, 1336 (1989)

44. X. Liou, W. Liou, Int. J. Theor. Phys 49(5), 1088 (2010)

45. S. Zhou, W. Liu, Mod. Phys. Lett. A 24(26), 2099 (2009)

46. M. Visser, Int. J. Mod. Phys. D 12, 649 (2003)

47. S.A. Hayward, Phys. Rev. D 49, 6467 (1994)

48. A. Ashtekar, B. Krishnan, Living Rev. Relativ. 7, 10 (2004)

49. C. Barcelo, S. Liberati, M. Visser, Living Rev. Relativ. 8, 12 (2005)

50. A. Vikman, Phys. Rev. D 71, 023515 (2005)

51. R.L. Mallett, Phys. Rev. D 31(2), 416 (1985)

52. A.C. Wall, Phys. Rev. D 85, 104049 (2012)

53. I. Brevik, S. Nojiri, S.D. Odintsov, L. Vanzo, Phys. Rev. D 70, $043520(2004)$

54. P.F. Gonzalez-Diaz, C.L. Siguenza, Nucl. Phys. B 697, 363 (2004)

55. D.H. Hsu, A. Jenskins, M.B. Wise, Phys. Lett. B 597, 270 (2004)

56. J.A.S. Lima, J.S. Alcaniz, Phys. Lett. B 600, 191 (2004)

57. H. Mosheni Sadjadi, Phys. Rev. D 73, 0635325 (2006)

58. S. Nojiri, S.D. Odintsov, Phys. Rev. D 70, 103522 (2004)

59. S. Nojiri, S.D. Odintsov, Phys. Lett. B 595, 1 (2004)

60. T. Jacobson, R. Parentani, Found. Phys. 33, 323 (2003)

61. H. Hadi, F. Darabi, Y. Heydarzade, EPL (Europhys. Lett.) 131(5), 59001 (2020)

62. H. Hadi, Y. Heydarzade, F. Darabi, K. Atazadeh, Eur. Phys. J. Plus 135(7), 1-14 (2020)

63. P. Rudra, R. Biswas, U. Debnath, Astrophys. Space. Sci. 354, 597 (2014) 